\title{
Role of vitamins on various biological aspects of an egg parasitoid, Trichogramma chilonis (Ishii) (Hymanoptera: Trichogrammatidae) on its laboratory host, Corcyra cephalonica
}

\author{
Kapinder $^{1}$, Tarkeshwar ${ }^{1}$ and Ashok Kumar Singh ${ }^{2}$ \\ 1, 1,2 (Department of Zoology, University of Delhi, India)
}

\begin{abstract}
Parasitic wasps play major role in sustainable agriculture through their ability to regulate population of herbivorous insect pests and nutrition is the key component to the success of the parasitoids. In the present study, the effect of nutrition was revealed by a significantly higher survival of both male and female parasitoids when fed on sucrose and other vitamins as compared with water fed and unfed. The survival of adult wasps was also not much affected when different concentration of vitamins was tested except for pyridoxine in which survival of adult wasp was inversely related to concentration. Female parasitoids lived longer than the male in all treatments as well as in control. Parasitisation by female T. chilonis showed no significant difference except pyridoxine which showed lowest parasitisation percentage. The sex ratio in all treatments showed female biased populations except pyridoxine which showed male biased population. The present study may be helpful to select the best artificial food source which can be artificially sprayed in the field to increase the longevity and parasitisation potential of $T$. chilonis
\end{abstract}

Keywords: Survival, Parasitisation, Trichogramma, Sex-ratio, vitamin, sucrose

\section{Introduction}

Host plant, its pest and parasitoid of pest form a triangle of interacting forces in which nutrition play a decisive role in influencing the pest parasitoid relationship. The overall performance of a parasitoid is largely dictated by their energy resources. Among all the insects, parasitoids are unique in their nutritional requirements as some adult parasitoids not only depend on the host for feeding, but may also require sugar solutions, such as nectar or honeydew, as a source of energy (Jervis and Kidd, 1986). Nutritional deficiency in parasitoids causes poor parasitization, emergence and poor sex ratio, resulting in their reduced efficiency as biological control agents.

Carbohydrates provide an important source of energy to parasitoids as in other animals. Sugar feeding has been reported to increase parasitoids longevity (Wäckers, 2001; Siekmann et al., 2001) as well as its fecundity (Schmale et al., 2001). In addition, well fed parasitoids are usually more active and more focused in seeking out their herbivores hosts (Wäckers, 1994). Therefore, the availability of sugar source is a key factor in the population dynamics of a parasitoid-host system in biological control program.

Although the sugars are likely to be the most important nutrients in promoting longevity, there is also the possibility that micronutrients such as vitamins may also benefit parasitoid. There is very little knowledge on the effects of dietary components, especially vitamins, on the longevity and reproduction of hymenopteran parasitoid especially Trichogramma spp. which prevent us from making any generalizations on these species. It is the most important and has received the maximum attention, because of its importance in biological control. Trichogramma has been attempted to control against at least 28 different phytophagous pest species on 20 different crops. In India, 12 indigenous species of Trichogramma have been used, besides a few introduced species in release programmes. Among these, Trichogramma chilonis Ishii, is widely distributed in the Indian subcontinent, which has been widely used for the control of many lepidopterous pests.

Present study was done to understand the role of some water soluble vitamins on the parasitisation, adult emergence, and sex-ratio of Trichogramma chilonis.

\section{Materials And Methods}

Trichogramma chilonis Ishii (Hymenoptera: Trichogrammatidae) was reared on rice meal moth, Corcyra cephalonica Stainton (Lepidoptera: Galleriidae). Initial culture of the host insect and the egg parasitoid was started from the insects obtained from the Biological control laboratory of the Division of Entomology, I.A.R.I., New Delhi.

\section{A. Host insect culture}

Larvae of C. cephalonica were reared on broken grains of maize (Zea mays), Jowar (sorghum vulgare) and Bajra (Pennisetum typhoides) that were sterilized in oven at a temperature of $100^{\circ} \mathrm{C}$ for $1 \mathrm{~h}$ to eliminate the 
possibility of infestation by other stored grain pests. All the grains were mixed thoroughly and put in $3 \mathrm{~L}$ glass jars and inoculated with freshly laid eggs of C. cephalonica at $0.5 \mathrm{cc}$ per jar. The culture was maintained at a temperature of $27 \pm 2^{\circ} \mathrm{C}, 55 \pm 5 \% \mathrm{RH}$ and a photoperiod of $12 \mathrm{~L}$ : 12D. Freshly emerged adult moths were collected every morning and transferred to mating cum oviposition cages. It consisted of an inverted funnel (21 $\mathrm{cm}$ dia X $23 \mathrm{~cm} \mathrm{ht}$ ) fitted over a sieve $(22 \mathrm{~cm} \mathrm{dia})$ having a mesh size of $10 \mathrm{mesh} / \mathrm{cm}$. The mouth of the stem of the funnel was covered with a cotton plug soaked with $10 \%$ sucrose solution as an adult feed. The adults were released from the stem of the funnel. The whole set up was placed over a tray lined with filter paper to collect the eggs that passed through the sieves. Use of sieve at the open end of the funnel ensured collection of clean eggs in the tray, devoid of any scales or broken parts of the adult moths. Eggs were collected daily and were used for further inoculation for maintaining the host culture, parasitoids multiplication and other studies.

For multiplication of $T$. chilonis and other studies, $0-24 \mathrm{~h}$ old host eggs were exposed to ultra violet radiation in a closed wooden chamber at a distance of $15 \mathrm{~cm}$ from the UV-lamp, for about 30min., in order to kill the developing host embryo.

\section{B. Egg parasitoid culture}

Culture of the egg parasitoid, T. chilonis, was maintained in the glass vials $(2.5 \mathrm{~cm}$ dia $\mathrm{X} 10 \mathrm{~cm} \mathrm{ht})$. The adult parasitoids were offered an egg card $(8 \mathrm{~cm} \mathrm{X} 2.5 \mathrm{~cm})$, made of Ivory paper, containing approximately 1000 sterilized healthy eggs of $C$. cephalonica. They were allowed to parasitize the eggs for $24 \mathrm{~h}$ so as to prevent superparasitism. Each vial was provided with 50\% honey solution as adult food in the form of fine streak on the inner wall of the glass vial and secured tightly with cotton plug. After $24 \mathrm{~h}$ the egg cards were removed and kept for development in a fresh tube after clearing the parasitoids. Parasitized eggs turned black on the fourth day after parasitisation. These parasitised egg cards were used for further maintenance of culture as well as for the experiment. The parasitoid culture was maintained in a B.O.D. incubator at $27 \pm 1^{\circ} \mathrm{C}$, at $65 \pm 5 \%$ R.H and a photoperiod of 12:12 (L: D).

\section{Preparation of the vitamin solutions}

The different concentrations of the vitamins were prepared by dissolving the required amount of the vitamin in $20 \%$ sucrose solution. The vitamins selected for the bioassay were ascorbic acid, riboflavin, pyridoxine, thiamine and cyanocobalamin. To prepare $0.1 \%$ test solution $10 \mathrm{mg}$ of the vitamin was dissolved in $10 \mathrm{ml}$ of $20 \%$ sucrose solution and $0.001 \%$ test solution was prepared by serial dilution of $0.1 \%$ concentration in $20 \%$ sucrose solution.

\section{Effect on longevity}

The adult male and female parasitoids were separated on the basis of antennal variation. Diet was provided in the form of fine streak along the glass wall twice a day. Longevity of the parasitoid was recorded on the basis of mortality of the parasitoid in each vial, after an interval of every $12 \mathrm{~h}$. The replicates were maintained at $26 \pm 1{ }^{\circ} \mathrm{C}$ temperature and $70 \pm 5 \%$ relative humidity with $12: 12$ of L: D photoperiod. The bioassays were replicated five times, each replicate consisting of responses of 10 wasps.

\section{E. Effect on parasitisation and sex-ratio}

One pair of adult $T$. chilonis was released in each homeopathic vial and provided different vitamin diets for two hour prior to placement of egg cards. Thereafter single egg card $(2.5 \mathrm{~cm} \mathrm{X} 0.5 \mathrm{~cm})$ containing $100 \mathrm{UV}$ sterilized eggs of $C$. cephalonica (pasted with diluted acacia gum), kept in each vial and females were allowed to parasitize the eggs for $24 \mathrm{~h}$. then the egg cards were removed from the vials and kept in B.O.D. incubator. The number of eggs parasitized was recorded by counting the blackened eggs. Total number of parasitoids emerged out of the parasitized eggs and sex ratio were recorded.

\section{F. Statistical analysis:}

Data were subjected to one-way ANOVA. Means were separated by Tukey's test to analyze the significant difference between the control and different concentrations and also differences among different concentrations. All statistical analysis was performed on computer software program SigmaStat 2.0 (Jandel Scientific, 1995). Whereas, sex-ratio was analyzed by student t-test.

\section{A. Effect on longevity}

\section{Results}

The longevity of male and female parasitoid, T. chilonis, fed on $20 \%$ sucrose solution (9.5 days and 10.9 days respectively) was significantly longer as compared to those fed on water (1.1 days) or unfed (0.8 days) parasitoids. Also, the survival of both male and female parasitoids on different vitamins was significantly higher than on water fed or starved parasitoids. However, no significant difference was observed in the survival of the 
male parasitoids fed on $0.001 \%$ concentration of different vitamins and $20 \%$ sucrose. Similar observations were also recorded in female parasitoids that had statistically equal survival on different vitamins at $0.001 \%$ concentration and $20 \%$ sucrose, except for pyridoxine (13.4 days) which showed significantly $(\mathrm{P}<0.05)$ longer survival than on all other vitamin treatments (Table.1).

The longevity of male parasitoids fed on $0.1 \%$ concentration of different treatments was statistically similar with $20 \%$ sucrose solution. The survival of male wasps was significantly lower on pyridoxine (7.4 days) as compare to ascorbic acid (10.9 days). There was no significant difference was observed in the survival of the female parasitoids fed on different vitamins ( $0.1 \%$ concentration) and control (20\% sucrose) (Table.1).

Table 1. Survival of $T$. chilonis on $20 \%$ sucrose solution mixed with different vitamins

\begin{tabular}{|c|c|c|c|c|c|}
\hline \multirow[t]{2}{*}{ Sex } & \multirow[t]{2}{*}{ Type of food } & \multicolumn{4}{|c|}{$\begin{array}{c}\text { Concentration (\%) } \\
\text { Mean } \pm \text { S.D. }\end{array}$} \\
\hline & & & $0.1 \%$ & & $0.001 \%$ \\
\hline \multirow{8}{*}{ Male } & No food & $0.8 \pm 0.274^{\mathrm{b}}$ & & $0.8 \pm 0.274^{\mathrm{b}}$ & \\
\hline & Water & $1.1 \pm 0.42^{\mathrm{b}}$ & & $1.1 \pm 0.42^{\mathrm{b}}$ & \\
\hline & Sucrose $(20 \%)$ & $9.5 \pm 1.06^{\mathrm{ac}}$ & & $9.2 \pm 2.52^{\mathrm{a}}$ & \\
\hline & Ascorbic acid & $10.9 \pm 0.55^{\mathrm{a}}$ & & $7.1 \pm 1.25^{\mathrm{a}}$ & \\
\hline & Riboflavin & $10.3 \pm 0.97^{\mathrm{ac}}$ & & $8.4 \pm 0.89^{\mathrm{a}}$ & \\
\hline & Pyridoxine & $7.4 \pm 0.65^{c}$ & & $9.9 \pm 2.10^{\mathrm{a}}$ & \\
\hline & Thiamine & $8.9 \pm 2.65^{\mathrm{ac}}$ & & $8.5 \pm 1.54^{\mathrm{a}}$ & \\
\hline & Cyanocobalamin & $8.4 \pm 2.966^{\mathrm{ac}}$ & & $7.8 \pm 0.57^{\mathrm{a}}$ & \\
\hline \multirow{8}{*}{ Female } & No food & $0.9 \pm 0.42^{\mathrm{b}}$ & & $0.9 \pm 0.42^{b}$ & \\
\hline & Water & $1.4 \pm 0.42^{\mathrm{b}}$ & & $1.4 \pm 0.42^{\mathrm{b}}$ & \\
\hline & Sucrose $(20 \%)$ & $10.9 \pm 1.34^{\mathrm{a}}$ & & $10.9 \pm 1.34^{\mathrm{ac}}$ & \\
\hline & Ascorbic acid & $13.2 \pm 4.56^{\mathrm{a}}$ & & $9.38 \pm 1.89^{\mathrm{a}}$ & \\
\hline & Riboflavin & $12.4 \pm 1.71^{\mathrm{a}}$ & & $10.4 \pm 0.96^{\mathrm{a}}$ & \\
\hline & Pyridoxine & $10.5 \pm 3.72^{\mathrm{a}}$ & & $13.4 \pm 2.41^{\mathrm{c}}$ & \\
\hline & Thiamine & $12.0 \pm 3.66^{\mathrm{a}}$ & & $10.4 \pm 1.43^{\mathrm{a}}$ & \\
\hline & Cyanocobalamin & $11.2 \pm 1.82^{\mathrm{a}}$ & & $9.8 \pm 0.45^{\mathrm{a}}$ & \\
\hline
\end{tabular}

Note: Means followed by different superscripts in the particular column differ significantly with in the $\operatorname{sex}(\mathrm{P}<0.05)$

A comparison was done between two concentrations $(0.1 \%$ and $0.001 \%)$ of tested vitamins, where the longevity of male parasitoids fed on $0.1 \%$ ascorbic acid and riboflavin was significantly higher than the wasps fed on $0.001 \%$. Whereas, survival on pyridoxine at $0.1 \%$ was statistically lower than $0.001 \%$ concentration. However, no significant difference was observed in the survival of female wasps when compared between $0.1 \%$ and $0.001 \%$ concentration of vitamins (Table 2 ).

Table 2. Mean survival of male and female $T$. chilonis on different concentrations of vitamin

\begin{tabular}{|c|c|c|c|c|c|c|}
\hline \multirow{2}{*}{ Sex } & \multirow[b]{2}{*}{ Conc. $(\%)$} & \multicolumn{5}{|c|}{ Survival (days) Mean \pm S.D. } \\
\hline & & Ascorbic acid & Riboflavin & Pyridoxine & Thiamine & Cyanocobalamin \\
\hline \multirow{2}{*}{ Male } & 0.001 & $7.1 \pm 1.25^{\mathrm{a}}$ & $8.4 \pm 0.89^{\mathrm{a}}$ & $9.9 \pm 2.1^{\mathrm{a}}$ & $8.5 \pm 1.54^{\mathrm{a}}$ & $7.8 \pm 0.57^{\mathrm{a}}$ \\
\hline & 0.1 & $10.9 \pm 0.55^{\mathrm{b}}$ & $10.3 \pm 0.97^{\mathrm{b}}$ & $7.4 \pm 0.65^{\mathrm{b}}$ & $8.9 \pm 2.65^{\mathrm{a}}$ & $8.4 \pm 2.97^{\mathrm{a}}$ \\
\hline \multirow{2}{*}{ Female } & 0.001 & $9.38 \pm 1.89^{\mathrm{a}}$ & $10.4 \pm 0.96^{\mathrm{a}}$ & $13.4 \pm 2.41^{\mathrm{a}}$ & $10.4 \pm 1.43^{\mathrm{a}}$ & $9.8 \pm 0.45^{\mathrm{a}}$ \\
\hline & 0.1 & $13.2 \pm 4.56^{\mathrm{a}}$ & $12.4 \pm 1.71^{\mathrm{a}}$ & $0.5 \pm 3.72^{\mathrm{a}}$ & $12.0 \pm 3.66^{\mathrm{a}}$ & $11.2 \pm 1.82^{\mathrm{a}}$ \\
\hline
\end{tabular}

\section{B. Effect on parasitisation and sex-ratio}

The parasitisation percentage was not significantly affected by any vitamins except pyridoxine. The parasitisation on ascorbic acid (68\%), riboflavin $(68.4 \%)$, thiamine $(67.8 \%)$ and cyanocobalamin $(66.5 \%)$ was statistically similar, whereas, pyridoxine showed significantly lower parasitisation of $33.6 \%$ when compare to rest of the vitamins.

The emergence trend of male and female parasitoids reared on different diets showed significant correlation between the type of food and sex of the progeny. There was no significant difference in male-female sex ratio was observed when parent parasitoids were fed pyridoxine (male $51.22 \%$ and female $41.47 \%$ ). However, when parent wasps were fed on other tested food, significantly higher numbers of female progeny was produced as compared to males. 
Table 3. Parasitisation percentage and sex-ratio of $T$. chilonis on $0.1 \%$ concentration of vitamins

\begin{tabular}{llcc}
\hline \multicolumn{1}{c}{ Food } & \multicolumn{1}{c}{$\begin{array}{c}\text { Parasitisation } \\
(\boldsymbol{\%})\end{array}$} & \multicolumn{1}{c}{$\begin{array}{c}\text { Mean } \pm \text { S.D. } \\
\text { Sex-ratio }\end{array}$} \\
\cline { 3 - 4 } & \multicolumn{1}{c}{ Male } & \multicolumn{1}{c}{ Female } \\
\hline 20\% Sucrose & $58.4 \pm 9.18^{\mathrm{a}}$ & $20.00 \pm 6.11^{\mathrm{a}}$ & $63.35 \pm 12.32^{\mathrm{b}}$ \\
Ascorbic acid & $68 \pm 4.69^{\mathrm{a}}$ & $25.29 \pm 12.58^{\mathrm{a}}$ & $63.30 \pm 5.57^{\mathrm{b}}$ \\
Riboflavin & $68.4 \pm 4.93^{\mathrm{a}}$ & $33.22 \pm 6.63^{\mathrm{a}}$ & $59.80 \pm 10.13^{\mathrm{b}}$ \\
Pyridoxine & $33.6 \pm 9.762^{\mathrm{b}}$ & $51.22 \pm 16.50^{\mathrm{a}}$ & $41.47 \pm 23.97^{\mathrm{a}}$ \\
Thiamine & $67.8 \pm 8.38^{\mathrm{a}}$ & $28.02 \pm 4.97^{\mathrm{a}}$ & $58.74 \pm 8.71^{\mathrm{b}}$ \\
Cyanocobalamin & $66.5 \pm 7.31^{\mathrm{a}}$ & $34 \pm 8.84^{\mathrm{a}}$ & $53.72 \pm 9.03^{\mathrm{b}}$ \\
\hline
\end{tabular}

Note: Means followed by different superscripts in the column of parasitisation and along the row in sex-ratio differ significantly $(\mathrm{P}<0.05$, t-test).

\section{Discussion}

The provision of supplemental food to parasitoid, Trichogramma chilonis appears to be an important factor for increasing longevity of the parasitoid. Both the male and female T. chilonis, fed on sucrose solution survived for longer duration as compared to those fed on water or starved wasp. Earlier studies have also shown that starvation significantly reduces the longevity of male and female parasitoids (Jervis and Kidd, 1986), whereas provisioning of food increases the longevity of $T$. carverae and $T$. nr brassicae (Gurr and Nichol, 2000). In the present study, T. chilonis has been observed to survive less than one day ( 0.9 days) without food. However another species of this parasitoid, T. brassicae and T. carverae have reported to survive without food for 5 days and 6 days respectively without food (Gurr and Nichol, 2000).

The longevity of the parasitoids on different vitamin treatments and control sucrose solution was statistically same. This indicates that the tested concentrations equally support survivorship of both male and female parasitoids. Individual sugars and mixture have been also earlier observed to significantly affect the longevity of parasitoid, Bathyplectes curculionis (Jacob and Evans, 2004). The survivorship of the parasitoids on different vitamins, except pyridoxine, was comparable with that of sucrose solution. Pyridoxine has concentration dependent effect on survival period of parasitoids. The observation clearly indicates that dose of vitamin is critical for the overall fitness of the parasitoids, and requirement of pyridoxine by $T$. chilonis is far less than other vitamins tested. Higher quantity of vitamin in diet has been reported to decrease the feeding of insects (Friend, 1958). Etebari and Matindoost (2004) observed that high dose of vitamin $\mathrm{B}_{3}$ in the diet interrupt feeding and normal growth of silkworm larvae. The observed High mortality was recorded during molting of larvae. Significant decrease in larval weight was observed when silkworm larvae were fed on $3 \%$ vitamin C incorporated diet. Incorporation of high level of ascorbic acid in house cricket diet has been observed also to inhibit spermatogenesis and decrease the viability of eggs (Mcfarlane, 1992).

Vitamins do not appear to influence parasitization percentage of insects, except pyridoxine that has been observed to reduce rate of parasitization. This may be because pyridoxine adversely affects egg production of parasitoids. However, there are certain reports that some vitamins such as Vitamin E enhance the egg production in the insects (House, 1966).

All vitamins, except pyridoxine, appear to cause female biased populations. This is contrary to the earlier observation of Leatemia et al. (1995), who found male biased population in long lived females of $T$. minutum when fed on different concentrations of honey solution. In the present study, the sex ratio was studied with the eggs, laid in $24 \mathrm{hr}$ of emergence, and not for the whole life of the parasitoid.

\section{Conclusion}

Vitamins play important role in survival, longevity and parasitization of T. chilonis. However, effect of pyridoxine on the fitness of this parasitoid is dose dependent. Lower dose of pyridoxine has positive effect but at higher dose it cause lower longevity, lower parasitization and male biased populations. This means that higher concentration of pyridoxine adversely affect fitness of $T$. chilonis. The reversed has been reported for riboflavin, which inhibits growth of several insect species at lower dose but this ill effect is not observed at higher dose (Akov, 1962; Vanderzant, 1963). However, to understand the actual effect of any component of food on the fitness of the parasitoids, studies 2 or 3 generations are required (Ouye and Vanderzant, 1964; Kasting and McGinnis, 1967; Dadd, 1973).

\section{Acknowledgements}

We thank University of Delhi, India, for providing facilities in the Department of Zoology, and Prof. K.N Saxena for his valuable guidance during this study. We gratefully acknowledge Research Fellowship provided by the University Grants Commission for this work. 


\section{References}

[1] M.A. Jervis and N.A.C. Kidd. Host feeding strategies in hymenopteran parasitoids. Biol. Rev., 1986, 61: $395-434$.

[2] F.L. Wäckers. A comparison of nectar and honeydew sugars with respect to their utilization by the hymenopteran parasitoid Cotesia glomerata. J. Insect Physiol., 2001, 47: 1077-1084.

[3] G. Siekmann, B.Tenhumberg and M.A. Keller. Feeding and survival in parasitic wasps: sugar concentration and timing matter. Oikos, 2001, 95: 425-430.

[4] I. Schmale, F.L. Wäckers, C. Cardona and S. Dorn. Control potential of three hymenopteran parasitoid species against the bean weevil in stored beans: the effect of adult parasitoid nutrition on longevity and progeny production. Biol. Cont., 2001, 21: 134-139.

[5] F.L. Wäckers. The effect of food deprivation on the innate visual and olfactory preferences in the parasitoid Cotesia rubecula. J. Insect Physiol., 1994, 40: 641-649.

[6] Jandel Sigma Stats. Computer software for statistical analysis, 1995.

[7] G.M. Gurr and H.I. Nicol. Effect of food on longevity of adults of Trichogramma carverae Oatman and Pinto and Trichogramma nr brassicae Bezdenko(Hymenoptera: Trichogrammatidae). Aus. J. Entomol., 2000, 39: 185-187.

[8] H.S. Jacob and E.W. Evans. Influence of different sugars on the longevity of Bathyplectes curculionis (Hymenoptera: Ichneumonidae). J. Appl. Entomol., 2004, 128(4): 316-320.

[9] W.G .Friend. Nutritional requirements of phytophagous insects. Annu. Rev. Entomol., 1958, 3: 57-74.

[10] K. Etebari, and L Matindoost. Effects of hypervitaminosis of vitamin B B $_{3}$ on silkworm biology. J. Biosci., 2004, 29(4): 417-422.

[11] J.E. Mcfarlane. Can ascorbic acid or beta- carotene substitute for vitamin E in spermiogenesis in the house cricket (Acheta domesticus). Comp. Biochem. Physiol. 1992, A103: 179-181.

[12] H.L. House. Effects of varying the ratio between the amino acids and other nutrients in conjunction with a salt mixture on the fly Agria affinis (Fall.). J. Insect Physiol., 1966, 12: 299-310.

[13] J.A. Leatemia, J.E. Laing, and J.E. Corrigan. Effects of adult nutrition on longevity, fecundity and offspring sex ratio of Trichogramma minutum Riley (Hymenoptera: Trichogrammatidae). Can. Entomol., 1995, 127: 245-254.

[14] S. Akov. A qualitative and quantitative study of the nutritional requirements of Ades aegypti larvae. J. Insect Physiol., 1962, 8: 319335 .

[15] E.S. Vanderzant. Nutrition of the bollweevil larva. J. Econ. Entomol., 1963, 56: 357-362.

[16] M.J. Ouye and E.S. Vanderzant. B vitamin requirements of the pink bollworm. J. Econ. Entomol., 1964, 57: 427-430.

[17] R. Kasting, and A.J. McGinnis. An artificial diet and some growth factor requirements for the pale western cutworm. Can. J. Zool., 1967, 45: 787-796.

[18] R.H. Dadd. Insect nutrition: current developments and metabolic implications. Annu. Rev. Entomol., 1973, 18: 381-420. 